\title{
Pensar raza: La Interseccionalidad un camino de desafíos y encrucijadas
}

Think Race: Intersectionality A Path of Challenges and Crossroads

\author{
Ivette Sóñora Soto' (iD 0000-0003-4111-9553
}

'Universidade Federal de Santa Catarina, Programa de Pós-Graduação em História, Florianópolis, SC, Brasil. 88.040-900-ppghst@contato.ufsc.br

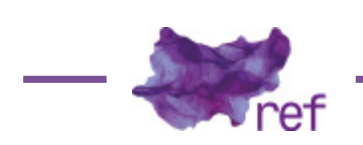

AKOTIRENE, Carla.

O que é interseccionalidade?

Belo Horizonte: Letramento, 2018.

\begin{abstract}
En O que é interseccionalidade? se expone la historicidad y al mismo tiempo el mapeo del concepto de interseccionalidad desde su emergencia, el fundamento y la entrada de este al espacio académico. Además, trae a colación las críticas realizadas al cuerpo teórico, lo cual la lleva a dialogar con feministas como Angela Davis, Patricia Hill Collins, Ochy Curiel, Houria Bouteldja, Oyérónkë Oywùmí, y las brasileras, Sueli Carneiro y Lélia Gonzalez, entre otras.

La autora tiene como objetivo colocar la interseccionalidad como herramienta teórica, metodológica y práctica que permita explicar y ver dentro del choque de las estructuras del racismo, del capitalismo y del cisheteropatriarcado la interacción simultánea de las arterias identitarias y las encrucijadas discursivas por las que atraviesan las mujeres negras. Dentro de estas avenidas - término que también es definido por Kimberle Crenshaw (2012) - están colocadas en una posición de vulnerabilidad con respecto a otros públicos. O sea se intenta denotar como dentro de este sistema interconectado de opresiones las mujeres negras están más expuestas porque están insertadas en más de una categoría de minoría social ya que pasan por situaciones de discriminación de forma diferente de otras personas - que en este caso hace referencia a las mujeres blancas, ya que solo hacen parte de una de esas categorías'.

Insistirá en este texto sobre la necesidad de pensar que existe un vínculo inseparable de manera estructural entre el racismo, el capitalismo y el cisheteropatriarcado y que las articulaciones decurrentes de esa cuestión conllevan a las mujeres negras a ser expuestas. También la otra cuestión implícita es el fracaso del feminismo blanco en socorrer a las "víctimas" negras porque dentro de sus análisis teóricos y propuestas están impregnado de racismo. El objetivo del libro también es destacar el aporte de las teóricas negras al establecer el concepto teórico de interseccionalidad dentro del campo académico, ya que las experiencias y reivindicaciones políticas no fueron tenidas en cuenta por el feminismo blanco al igual que los movimientos
\end{abstract}

\footnotetext{
' Este criterio lo manejará en varias de las entrevistas realizadas por motivo de la salida de esta obra: GAMA, Luana. "'Conhecimento precisa respeitar toda diversidade', diz Carla Akotirene na UFBA". Agenda Arte e Cultura. 2018. Disponível em: https://www.agendartecultura.com.br/noticias/conhecimento-precisa-respeitar-diversidade-carlaakotirene-ufba/. Acceso en: 05/02/2019. MENEZES, Jamile. \#FalaPreta - Carla Akotirene fala de seu livro na coleção "Feminismos Plurais", de Djamila Ribeiro!. Portal Soteropreta. 2018. Disponible en: http://portalsoteropreta.com.br/ falapreta-carla-akotirene-fala-de-seu-livro-na-colecaofeminismos-plurais-de-djamila-ribeiro/. Acceso en: 05/02/2019.
} 
antirracistas ya que este solo se enfocaría en los hombres negros, lo cual demuestra que la lucha de los negros tampoco escapa al carácter machista y al régimen heterosexista.

¿Qué es interseccionalidad? Pregunta que da título al libro y que al mismo tiempo lleva por muchos caminos, abre un abanico de cuestiones, de reconocimientos, de críticas y propuestas. Carla Adriana da Silva Santos, más conocida por Carla Akotirene², será la guía por el laberinto de la escritura ¿Qué es la interseccionalidad? Y la respuesta comienza con la misma Carla Akotirene, lideresa Negra, nordestina. Bachiller en Servicio Social, Maestra y doctoranda en Estudios Interdisciplinares de Género, Mujeres y Feminismos por la Universidad Federal de Bahía (UFBA). Adopta el nombre de guerra, Akotirene, para enfrentar el poder colonial, racial hetorosexista y al mismo tiempo ella misma se rescata junto a su ancestralidad.

Para la autora, representar a las mujeres negras nordestinas en el espacio académico fue el honor concedido, por integrar con este volumen la "Coleção Feminismos Plurais", coordinado por la filósofa y feminista Djamila Ribeiro. Ya que desde la práctica trabaja como asistente social atendiendo víctimas de la violencia doméstica en el municipio de Salvador/BA. Oportunidad que se le presentó y que fue muy bien aprovechado para destacar la importancia del conocimiento que es trasmitido por las mujeres negras sobre todo de aquellas que no se encuentran dentro del espacio académico. Pues, como bien plantea, "O conhecimento precisa respeitar toda diversidade que representa o feminismo negro" 3 (BIKONOTICIAS, 2010). Es por tanto su objetivo presentar la raíz política, los principios básicos y las disonancias y sobre todo los desafíos que vendrán o que están.

El volumen constituye el primer libro autoral de Carla Akotirene que tiene como hilo conductor el concepto de interseccionalidad, la referencia a la autora que lo acuñó, y el Atlántico como locus que dio la posibilidad de establecer todas las interconexiones que denuncia esta noción desde lo teórico y la praxis. También forman parte los agradecimientos, las notas y las referencias bibliográficas empleadas, y con las escritas de referencia en el campo académico; prólogo de la doctora en sociología Ângela Figueiredo en la solapa. Y nota introductoria de la doctora en Estudios Étnicos y Africanos Zelinda Barrios, en la contracubierta del libro.

En principio, Carla Akotirene (2018) parte que el concepto vertebrador es una sensibilidad analítica pensada por las feministas negras, cuyas reivindicaciones intelectuales y experiencias estaban marcadas por el silencio, el olvido y el hecho de desconocerlo por parte del movimiento feminista blanco y antirracista. La primera ruta fue buscar la memoria histórica del concepto y para ello era necesario cruzar el Atlántico y hacer la primera parada en Estados Unidos para comenzar el camino teórico.

Desde la memoria, parte para situar la emergencia del concepto de la crítica realizada por las feministas negras a las leyes contra la discriminación. Acuñado por Kimberle Crenshaw, quien detenta que la interseccionalidad comienza por instrumentalizar teórica y metodológicamente a la inseparable estructura formada por el racismo, el capitalismo y el cisheteropatriarcado, que van a multiplicar las avenidas identitarias donde las mujeres negras son repetidamente alcanzadas por el cruzamiento y superposición del género, la raza y la clase como parte de los modernos aparatos coloniales.

El punto de partida de Akotirene es explicar porque es importante e imperativo asumir dentro de los activismos sociales y teóricos la existencia de una matriz colonial moderna, cuyas relaciones de poder son superpuestas en múltiples estructuras dinámicas; todas siendo merecedoras de atención política. Esas estructuras combinadas con la matriz colonial; primero, facilita que se instrumentalice conceptualmente raza, clase, nación y género, por tanto requiere de grupos victimizados. En segundo, provoca la sensibilidad interpretativa de los efectos identitarios y; por último, propone centrar la atención global a la matriz colonial moderna, para evitar cualquier posible desvío analítico, limitado para apenas un eje de opresión. Centrarse en este camino es la propuesta para la lectura.

Respecto a la cuestión de las avenidas identitarias, primero se parte en reconocer como el racismo estructural está unido inseparablemente al capitalismo y al sexismo; y como dentro de la matriz de opresiones la mujer negra es alcanzada. Por tal motivo, Akotirene - en movimiento semejante al de Kimberle Crenshaw - pone la atención en el lugar ocupado por las mujeres negras, espacio donde convergen esas opresiones y donde estas son mayormente afectadas en relación con otros grupos. Por la misma razón ella plantea que se hace necesario y muy oportuno descolonizar las perspectivas hegemónicas sobre la teoría de la interseccionalidad y asumir el Atlántico como ese centro de opresiones cruzadas, al instituirse en el único que puede traducir la historia de la migración forzada de africanas y africanos como mercancías; y también pensarlo como la cura a las heridas coloniales ocasionadas por Europa, a las culturas ahogadas, a los binarismos identitarios, a la contraposición de humanos y no humanos.

${ }^{2}$ Carla Adriana da Silva Santos adoptaría Akotirine como su nombre político durante la primera sesión del proyecto POMPA (Mentes y Puertas Abiertas en el Instituto Steve Biko).

3 "El conocimiento necesita respetar toda la diversidad que representa el feminismo negro". 
Y es interesante verlo desde esta perspectiva, que como bien dice Isabel Hofmeyr, es el espacio de "emergência do capitalismo moderno enquanto sistema transnacional" ${ }^{4}$ (Isabel HOFMEYR, 2018, p. 12). Más adelante, reafirma conjuntamente en palabras de C. L. R. James que "os escravos tornam-se os primeiros indivíduos modernos"5 (apud HOFMEYR, 2018, p. 12). Y a pesar de ser concebida como esa modernidad donde su traducción es una complejidad de varias tradiciones convertida en algo nuevo, sin embargo, Ntongela Masilda va apuntar el olvido de la tradición africana en esa traducción (apud HOFMEYR, 2018, p. 12-13). Akotirene, basada en la profecía yoruba, le sugiere a la diáspora negra buscar sus caminos discursivos con atención a los acuerdos establecidos con los antepasados.

Ahora bien, su libro es una sistematización, la historicidad del concepto le permitirá traer a otras feministas negras en la misma posición de importancia Lélia Gonzalez, Patricia Hill Collins, Audre Lorde, Achille Mbembe, Conceição Evaristo, entre otras. Pero Akotirene invocará a la mestiza que también sabe que su lucha es feminista porque enfrenta igualmente una lucha de carne, de fronteras, una guerra interior. Se reconoce como un producto de transferencia de valores culturales y espirituales de un grupo para otro, tricultural, monolingüe, bilingüe, multilingüe, asimismo se enfrenta con el dilema de las razas hibridas, ya que no sabe a qué colectividad pertenece al ser hija de una madre de piel oscura (Gloria ANZALDÚA, 2005, p. 705). Este mestizaje será convertido también en un punto de encuentro, de encrucijada dentro de las avenidas identitarias.

Para analizar y criticar al marxismo desde la articulación de las estructuras del racismo, capitalismo, cisheteropatriarcado y etario, Akotirene confronta con los teóricos, el discurso que pronunció Sojourner Truht en 1851, Las mujeres exigen la igualdad con los negros, en el primer Aniversario de la Asociación Americana para la lgualdad de Derechos que tuvo lugar en la Iglesia de los Puritanos donde exponía y demostraba como la experiencia de las mujeres negras fueron omitidas y nadie se preocupaba por los derechos de esta (Sojourner TRUHT, 2012, p. 62). La autora contrapone este criterio con los de Karls Marx y Fedérico Engels, quienes en su análisis del capitalismo no tuvieron en cuenta las víctimas no occidentales. Esta misma perspectiva ha sido mantenida por el feminismo blanco de izquierda; y es ahí que Akotirene cuestiona las metodologías y las interpretaciones que mantiene fuera de sus cuestionamientos y respuestas a las mujeres negras y por tal motivo sostiene una dependencia epistemológica asustadora de la Europa occidental y de Estados Unidos.

Otro asunto que interesa destacar del libro es su criterio sobre el cual se posiciona para decir claramente que la interseccionalidad exige orientación geopolítica y que Orí lleva a dialogar con las epistemologías del Sur, a partir de que las mismas intelectuales y feministas negras dentro de Estados Unidos responden a los saberes periféricos pues también sufren de racismo y sexismo epistémicos porque reflejan experiencias poscoloniales en las aguas atlánticas como las hermanas del otro lado de América. Aquí me quiero detener en las intelectuales y feministas negras como Lélia Gonzalez; en mi opinión hubiera sido valioso profundizar en la teoría de Améfrica Ladina, propuesta por esta autora con la cual critica el monocultarismo epistémico de los Estados Unidos (Lélia GONZALEZ, 1988); pues, quizás contraponer estos dos conceptos ayudaría a comprender mejor la realidad de las mujeres negras en Brasil y en el resto del Sur Global.

Con una mirada decolonial, Akotirene considera contraproducente emplear la teoría interseccional para localizar discriminaciones y violencias institucional contra indígenas, inmigrantes, mujeres, negros, religiosos del candomblé, gordos o grupos identitarios diversificados como Lésbicas, Gays, Bisexuales, Transexuales, Queer e Intersexos (LGBTQl); por tal motivo, les sugiere alfabetizarse con el feminismo negro que dialoga simultáneamente entre y con las avenidas identitarias del racismo, cisheteropatriarcado y el capitalismo. Sin embargo, en este punto de la discusión también hubiera hecho falta la indicación de la metodología para que estos grupos pudiesen apropiarse y pudieran saciar el hambre histórica de justicia social y política, sobre todo las mujeres indígenas.

Con el uso del concepto de interseccionalidad, Akotirene coloca nuevas cuestiones sobre el funcionamiento social, ideológicas y el poder. En este punto critica la apropiación intelectual indebida y mal acostumbradas de las academias europeas, de los estudios de blanquitudes brasileras, asimismo los órganos consultivos de igualdad y de control social de las naciones adoptasen políticas públicas, acumulativas, transversales y constitutivas de supuesto carácter interseccional y antidiscriminatorios. Dentro de esas prácticas se acostumbran usar la interseccionalidad como sinónimo de minorías políticas o diversidades, lo cual implica cuestionar la agencia de las mujeres negras y como si la encrucijada fuera el lugar de decisión de estas como víctimas: ¿es levantarse o continuar en el suelo, derrumbada? ¿Sentir o no las heridas de la

\footnotetext{
4 "emergencia del capitalismo moderno en cuanto sistema transnacional".

5 "los esclavos se tornaron los primeros individuos modernos".

"El Orisha Ori representa una deidad de la mitología yoruba, considerado un Orisha que rige todo lo referentes a los dioses santeros, así como los destinos de los humanos. Está relacionado con la espiritualidad de cada ser humano, y está incorporado en su espiritualidad, como una especie de Orisha personal, y está ligado a cada individuo desde su nacimiento.
} 
colonización? Por eso, la autora enfatiza: "É da mulher negra o coração do conceito de interseccionalidade"7 (AKOTIRENE, 2018, p. 19). Para también avisar de los peligros de vaciar del contenido real del concepto al ser asumido por los estudios académicos occidentales del Norte global y por eso no concuerda con el uso de feminismo interseccional ya que no reconoce el paradigma afrocéntrico en su concepción, pues al negarse se está cometiendo epistemicidio y racismo epistémico.

La otra cuestión que me parece interesante destacar es el diálogo que establece con las intelectuales africanas, entre ellas, Oyèronké Oyèwúmi, responsable por criticar la tendencia dominante de las caracterizaciones occidentales del mundo social, las cuales tienden a ser unilineal y universalista y por tal motivo excluyentes de agencias de muchas otras experiencias como los pueblos yorubas (Oyèronké OYìùMí, 2004, p. 1-3). Sus estudios a su vez demostraron que el pensamiento africano no era tenido en cuenta en las teorías sobre condición humana al considerar a África por largo tiempo como un continente misógino y primitivo. Su estudio sobre la familia yoruba demostró que la cuestión de género fue adicionada recientemente porque esta sociedad no se organizó en torno a las marcas de género, por tanto, concluye que el género no es socialmente construido sino también histórico. Por tal motivo, sus investigaciones se mueven alrededor del propósito de colocar en el escenario teórico las experiencias africanas para la constitución del conocimiento (OYìù̀Mí, 2004, p. 3).

Y este análisis le permitió a Carla Akotirene concluir que es necesario prestar atención a los anacronismos históricos sobre mujeres africanas después de la colonización. Por eso es importante remarcar la profecía yoruba de volver a los caminos discursivos en los acuerdos establecidos con los antepasados africanos. Y eso me recuerda a José Martíi que, en su ensayo filosófico y político "Nuestra América", planteará lo mismo, que la historia, el pensamiento que debe enseñarse de verdad y profundamente debe partir de nuestras experiencias arraigada en nuestras historias indígenas, aunque la de las Europa no se enseñé: "Nuestra Grecia es preferible a la Grecia que no es nuestra" (José MARTí, 1991, p. 18).

Esta cuestión del Sur global y África, de volver a ellas para entender nuestras realidades y experiencias, es fundamental para no repetir los errores del esencialismo y la universalización occidental definidos por la experiencia del Norte global; pues, aunque existe dentro de las universidades los estudios africanistas, los saberes derivados de sus vivencias son excluidos. Es por ello que el historiador Robert W. Slenes (2010, p. 29) plantea categóricamente que tanto los estudios sobre África y la diáspora como los/as profesores/as e investigadores/as negros/as deben tener una presencia destacada en las universidades brasileras; lo que converge con los criterios de Carla Akotirene sobre los estudios de interseccionalidad.

Visto de esta perspectiva y que resulta muy coherente puesto que permite aclararnos que es necesario aplicar la interseccionalidad a las políticas públicas - que es la forma de recalcar la discriminación que sufren las mujeres negras - y aquí es el caso específico de la Ley Maria da Penha ${ }^{9}$ que le da la espalda a las víctimas negras de violencia doméstica; al dicha ley no tener definido contornos interseccionales, conlleva a una situación de total vulnerabilidad a las mujeres negras.

Es por tal motivo que Akotirene enfatizará que la interseccionalidad es, ante todo, un lente analítico sobre la interacción estructural en sus efectos políticos y legales. La interseccionalidad nos muestra, hace visible como y cuando las mujeres negras son discriminadas y están mucho más expuestas y posicionadas en avenidas identitarias que las vuelven más vulnerables dentro del entrecruzamiento de las estructuras y flujos modernos.

Ahora bien, hay dos aspectos que aún me interesa destacar dentro de su obra y es el hecho de transgredir las fronteras del Norte Global y pensar su análisis desde lo global, su postura es una crítica al universalismo y esencialismo del feminismo blanco. También considero que es un libro muy denso para pensar la interseccionalidad, aunque aportó al debate las críticas realizadas desde el mismo feminismo negro. Pienso que le faltó un diálogo más profundo desde la interseccionalidad con el pensamiento mestizo, con el decolonial, pero sobre todo el intercambio más fluido con las feministas negras brasileiras que como Lélia Gonzalez ya tenía definido esta cuestión desde el Sul Global.

De todas formas, la interseccionalidad como concepto teórico tiene un camino por delante de desafíos y militancia. Y O que é interseccionalidade? consigue demostrar que ese es realmente una muy buena herramienta teórica y metodológica porque facilita visualizar la red y trampa de

\footnotetext{
7 "Es de la mujer negra el corazón del concepto de interseccionalidad".

${ }^{8}$ José Martí (1853-1995) fue el poeta e intelectual cubano que organizó la Guerra Necesaria para darle la independencia a Cuba; además del creador del Partido Revolucionario Cubano.

${ }^{9}$ Ante la violencia sufrida por la biofarmacéutica Maria da Penha Fernades a manos del esposo que intentó por dos ocasiones asesinarla, dejándola parapléjica y quedar impune, la Corte Interamericana de Derechos Humanos señaló como responsable del suceso al Gobierno de Brasil por no actuar ante situaciones como esta. Brasil para dar respuesta promulgó La Ley Maria da Penha: Ley 1 1.340/2006, la cual abarca la previsión de implementación de políticas para cohibir la violencia doméstica y familiar contra mujeres.
} 
las estructuras del racismo, el capitalismo y el cisheteropatriarcado con la consecuente interacción simultanea de las avenidas identitarias. No obstante, considero - y es una invitación amplia, abierta y sobre todo desprejuiciada para poder disfrutar - una buena escritura. Y hago mía, las palabras compartidas por Carla Batista: "Carla Akotirene es una de las personas que producen conocimientos que precisan ser compartidos, comprendidos y asimilados para que el mundo sea mejor. Buena lectura, comparta. Y lea el libro" (BATISTA, 2018).

\section{Referencias}

AKOTIRENE, Carla. O que é interseccionalidade? Belo Horizonte: Letramento, 2018.

ANZALDÚA, Gloria. "La conciencia de la mestiza/Rumo a uma nova consciência". Revista Estudos Feministas, Florianópolis, v. 13, n. 3, p. 704-719, 2005. Disponible en: http://www.scielo.br/pdf/ref/ v13n3/a15v13n3.pdf. Acceso en: 04/02/2019.

BATISTA, Carla. O que é Interseccionalidade? Folha PE, 07/09/2018 às 17h21, atualizado em 07/09/ 2018 às 17h21. Disponible en: https://www.folhape.com.br/noticias/noticias/mulheres-emmovimento/2018/09/07/NWS, 80564,70,1055,NOTICIAS,2190-O-QUE-INTERSECCIONALIDADE.aspx. Acceso en: 05/02/2019.

BIKONOTICIAS. "Bikodisse Entrevista: Carla Akotirene", Instituto Cultural Steve Biko, 08/07/ 2010. Disponible en: http://blogdabiko.blogspot.com/2010/07/bikodisse-entrevista-carla-akotirene.html. Acceso en: 06/02/2019.

CRENSHAW, Kimberle. "Cartografiando los márgenes. Interseccionalidad, políticas identitarias, y violencia contra las mujeres de color". In: PLATERO, Raquel (Lucas). Ed. Intersecciones: cuerpos y sexualidades en la encrucijada. Temas contemporáneos. España: Edicions Bellaterra, S.L, 2012, p. 87-122.

GONZALEZ, Lélia. "A categoria político-cultural de amefricanidade". Tempo Brasileiro. [Online]. Rio de Janeiro, n. 92/93, jan.-jun. 1988, p. 69-82.

HOFMEYR, Isabel. "O Atlântico Negro encontra o Oceano Índico: Forjando novos paradigmas do transnacionalismo para o Sul Global - Perspectivas literárias e culturais". Remates de Males [Online], Campinas-SP, v. 38, n. 1, p. 10-41, jan.-jun. 2018. Disponible en: https://periodicos.sbu.unicamp.br/ ojs/index.php/remate/article/view/8651431. Acceso en: 07/02/2019.

MARTí, José. "Nuestra América". In: MARTí, José. Obras Completas: Nuestra América, volumen 6. La Habana: Editorial Ciencias Sociales, 1991. p. 15-23.

OYìùMí, Oyèrónké. Conceitualizando o gênero: os fundamentos eurocêntricos dos conceitos feministas e o desafio das epistemologias africanas. CODESRIA Gender Series. Volume 1, Dakar, CODESRIA, 2004, p. 1-8.

SLENES, Robert Wayne Andrew. "A importância da África para as Ciências Humanas". História Social. Racismo: História e Historiografia [Online], n. 19, p. 19-32, 2010. Disponible en: https:// www.ifch.unicamp.br/ojs/index.php/rhs/article/view/314. Acceso en: 11/02/2019.

TRUHT, Soujourner. "Sufragio femenino". In: TRUTH, Sojourner et al. Feminismos Negros. Una Antología. Mercedes Jobardo (Introdución y notas). M3o Miquel, Ana Méndez, Marta García de Lucío, Sergio Ojeda y Esperanza Mojica (Traducción). España: Traficante de Sueños, 2012, p. 61-69.

Ivette Sóñora Soto (ivettesotocuba@gmail.com) posee el título de Maestría en Cultura Latinoamericana por el Instituto Superior del Arte (2006). Tiene experiencia en el área de Historia, actuando principalmente en temas de género, feminismo, subjetividades y movimientos sociales. Vinculada a la Línea de investigación de Historias entrecruzadas de subjetividades, género y poder, realiza doctorado en el Programa de Pos graduación en Historia en la UFSC e integra el grupo de investigadores del Laboratorio de Estudios de Género e Historia de la UFSC. 
COMO CITAR ESSE ARTIGO DE ACORDO COM AS NORMAS DA REVISTA

SÓÑORA SOTO, Ivette. "Pensar raza: La Interseccionalidad un camino de desafíos y encrucijadas". Revista Estudos Feministas, Florianópolis, v. 28, n. 1, e64872, 2020.

\section{CONTRIBUIÇÃO DE AUTORIA}

Não se aplica.

\section{FINANCIAMENTO}

O presente trabalho foi realizado com apoio da Coordenação de Aperfeiçoamento de Pessoal de Nível Superior - Brasil (CAPES) - Código de Financiamento 001 (Este trabajo fue realizado con el apoyo de la Coordinación de Perfeccionamiento del Personal de Nível Superior - Brasil (CAPES) - Código de Financiamento 001)

\section{CONSENTIMENTO DE USO DE IMAGEM}

Não se aplica.

\section{APROVAÇÃO DE COMITÊ DE ÉTICA EM PESQUISA}

Não se aplica.

\section{CONFLITO DE INTERESSES}

Não se aplica.

\section{LICENÇA DE USO}

Este artigo está licenciado sob a Licença Creative Commons CC-BY International. Com essa licença você pode compartilhar, adaptar, criar para qualquer fim, desde que atribua a autoria da obra.

\section{HISTÓRICO}

Recebido em 27/04/2019

Aprovado em 12/06/2019 